\title{
The effect of bioactive complex of quercetin, selenium, catechins and curcumin on cardiovascular risk markers in healthy population after a two month consumption
}

\author{
Madaric $\mathrm{A}^{1}$, Kadrabova $\mathrm{J}^{1}$, Krajcovicova-Kudlackova $\mathrm{M}^{1}$, Valachovicova $\mathrm{M}^{1}$, Spustova $\mathrm{V}^{1}$, \\ Mislanova $\mathrm{C}^{1}$, Kajaba $\mathrm{I}^{1}$, Blazicek $\mathrm{P}^{2}$
}

Slovak Medical University, Bratislava, Slovakia. jana.kadrabova@szu.sk

\begin{abstract}
Background: Nutrition is an important factor in prevention of degenerative age-related diseases. Health benefits of the functional food - cereal selenized onion biscuits with bioactive complex such as selenium in organic form, quercetin (onion), curcumin (curcuma) and catechins (green tea) were evaluated.

Methods: In a group of randomly selected 50 apparently healthy men, aged 30-50 years, the levels of total cholesterol, HDL-cholesterol, C-reactive protein (hs-CRP), homocysteine (HCy) and its nutritional determinants (methionine, vitamin B12, folic acid, cysteine, vitamin B6) and asymmetric dimethylarginine (ADMA) were measured and the LDL cholesterol and atherogenic index was calculated before and after a 2-month consumption period and after a 2-month wash-out period.

Results: The significant reduction of total cholesterol, LDL-cholesterol, atherogenic index, HCy and ADMA was found after onion biscuit consumption. Alternative pathway for HCy degradation using betaine as methyl donor is probably a sole argument for reduced $\mathrm{HCy}$ value at the significantly reduced concentrations of the methionine, folic acid, cysteine and vitamin B6. Value of hs-CRP was non-significantly reduced after biscuit consumption. Conclusion: The results of improved lipid profile, significantly reduced values of $\mathrm{HCy}$ and ADMA document a beneficial effect of cereal biscuit with selenized onion, curcuma and green tea in prevention of cardiovascular disease (Tab. 2, Ref. 19). Full Text in PDF www.elis.sk.

Key words: lipid profile, homocysteine, asymmetric dimethylarginine, cereal biscuit with selenized onion, green tea and curcuma.
\end{abstract}

One possibility for the prevention of chronic degenerative diseases is an increased intake of bioactive compounds with enhanced antisclerotic, antioxidative and antiinflammatory properties. Development and production of functional foods on base of bioactive compounds in plant sources can be a correct way. Functional foods is defined as food or food ingredients, which have the health beneficial effects related to prevention of civilization diseases. Plant kingdom is rich in natural sources of bioactive compounds such as polyphenols (mainly flavonoids such as flavonols, catechins and phytoestrogens), vitamins, mineral compounds, fibers with favourable effects on genetic, cellular, biochemical and physiological functions of the organism (1).

The functional food - cereal selenized onion biscuits with bioactive complex such as selenium in organic form, quercetin, curcumin, catechins and others presented in selected natural plant

${ }^{1}$ Slovak Medical University, Bratislava, Slovakia, and ${ }^{2}$ Alpha Medical, Bratislava, Slovakia

Address for correspondence: A. Madaric, PhD, Slovak Medical University, Limbova 12, SK-833 03 Bratislava 37, Slovakia.

Acknowledgement: This study was supported by the project of Ministry of Health of the Slovak Republic No. 2006/17-SZU-12. sources - selenized onion, curcuma and green tea was developed, produced and evaluated for health benefits.

\section{Subjects and methods}

The randomly selected group of 50 apparently healthy men ranged 30-50 years from Bratislava and surroundings was included in this intervention study of evaluation of health effects of cereal selenized onion biscuits consumption. The average BMI of $26.5 \pm 0.5 \mathrm{~kg} / \mathrm{m}^{2}$, WHR $0.887 \pm 0.008, \%$ of body fat $23.1 \pm 0.8$, blood pressure $138 \pm 2 / 83 \pm 1 \mathrm{~mm} \mathrm{Hg}$ (mean \pm SEM) were measured or calculated. The volunteers consumed $100 \mathrm{~g}$ of biscuits daily during 2 month. They were informed that their usual nutritional habits during biscuits consumption should not be changed. The Regional Ethic Committee approved the study, and all participants gave their written informed consent.

Composition of biscuits is described in the Table 1. Onion was selenium fortified by watering of onion fields with selenized water in the form of sodium selenan. Dried onion together with green tea and curcuma as the main natural sources of bioactive compounds were used for the production of biscuits, which was done by a common bakery technology in Pastry Factory, Liptovsky Hradok, Slovakia. Oat, rye and wheat whole-grain flour 
Tab. 1. Natural sources of bioactive compounds, energy value, macronutrients, micronutrients and fibre in $100 \mathrm{~g}$ of biscuit.

\begin{tabular}{lc}
\hline Nutritional compounds and nutrients & Amount \\
\hline Dried selenized onion $(\mathrm{g})$ & 6.0 \\
Green tea $(\mathrm{g})$ & 2.0 \\
Curcuma $(\mathrm{g})$ & 1.3 \\
Selenium $(\mathrm{g})$ & 115.0 \\
Energy $(\mathrm{kcal} / \mathrm{kJ})$ & $474 / 1983$ \\
Proteins $(\mathrm{g})$ & 11.2 \\
Saccharides $(\mathrm{g})$ & 52.4 \\
Fibre $(\mathrm{g})$ & 19.7 \\
Lipids $(\mathrm{g})$ & 24.4 \\
Saturated fatty acids $(\mathrm{g})$ & 14.6 \\
Monounsaturated fatty acids $(\mathrm{g})$ & 7.1 \\
Oleic acid $(\mathrm{g})$ & 6.73 \\
Polyunsaturated fatty acids $(\mathrm{g})$ & 2.70 \\
Linoleic acid $(\mathrm{g})$ & 2.46 \\
alpha-linolenic acid $(\mathrm{g})$ & 0.19 \\
\hline
\end{tabular}

was used as rich sources of fiber. Fat part of biscuits contained plant fat with low trans fatty acids and linen seed. Biscuits sample met the sensory requirements. Biscuits taste was finely salty, with characteristic onion aroma and more expressed seasoned taste and aroma. Biscuits fragility was adequate. The biscuits were analyzed on selected nutrients in the State Veterinary and Food Institute, at the accredited laboratory in Dolny Kubin, Slovakia.

Blood samples were taken after an overnight fasting using a standard procedure. EDTA was used as an anticoagulant. Serum concentrations of total and HDL-cholesterol were measured by standard laboratory methods. Value of LDL-cholesterol was calculated according to the Friedewald formula: LDL-cholesterol $=$ total cholesterol - triacylglycerols $/ 2.2-$ HDL-cholesterol. The atherogenic index $=$ LDL-cholesterol/HDL-cholesterol. The levels of total homocysteine ( $\mathrm{HCy}$ ), methionine and cysteine in plasma were measured by HPLC method with coulometric detection (2). Folic acid and vitamin B12 concentrations in serum were detected by electrochemiluminiscence immuno assay using the Roche test ECLIA. Serum levels of vitamin B6 were determined by HPLC with Chromsystems test. Asymmetric dimethylarginine (ADMA) levels in plasma were measured by the ELISA test from Imunodiagnostika company.
The intakes of vitamins, mineral and trace elements only in natural form were allowed (including biscuit consumption). The consumption of nutritional or pharmaceutical additives was excluded. The study was conducted in the late autumn and winter. The pair t-test was used to compare the results before consumption of biscuits and after the 2 month consumption or 2 month washout periods, and the results after consumption and at the 2 month washout period.

\section{Results and discussion}

A sufficient intake of food rich in dietary fibre is associated with a lower risk of cardiovascular diseases; the soluble and insoluble fibres reduce plasma total and LDL-cholesterol. Analysis of 10 prospective studies (using more than 336000 subjects) in USA and Europe has shown that an increase of fibre consumption by each $10 \mathrm{~g} /$ day was associated with $14 \%$ reduction (relative risk $\mathrm{RR}=0.86$ ) of all coronary events and with $27 \%$ decrease $(R R=0.73)$ of risk of coronary death (3). In addition to unsaturated fat and fibre, there are components of plant food that have the ability to reduce cardiovascular risk (antioxidant nutrients, selenium, polyphenols - mainly flavonoids such as flavonols/ quercetin/ and catechins).

Flavonoids exhibit their biological activities, including antiallergic, antiinflammatory, antiviral, antitumor and vasodilating actions. A sufficient intake of introduced bioactive compounds or their presence in functional foods can contribute to active protection against chronic degenerative diseases. Tea is one of the richest sources of flavonoids - catechins. The most important catechins are the following: epicatechin, epicatechin-3-gallate, epigallocatechin and epigallocatechin-3-gallate. The last named indicates the highest biological effects, such as antioxidative, antimutagenic, anticarcinogenic.

Flavonols, and specially quercetin exert endothelium-independent vasodilator effects, platelet antiagregant effects, inhibition of LDL oxidation, reduction of adhesion molecules and other inflammatory markers and prevention of neuronal oxidative and inflammatory damage (4). Quercetin produces undisputed antihypertensive and antiatherogenic effects, prevents endothelial dysfunction

Tab. 2. Total, HDL-, LDL-cholesterol, atherogenic index, homocysteine, its nutritional determinants, asymmetric dimethylarginine.

\begin{tabular}{|c|c|c|c|}
\hline Parameters & Before consumption (1) (2) (3) & 2-month after consumption & After 2-month wash-out \\
\hline Total cholesterol (mmol/1) & $5.17 \pm 0.14$ & $4.96 \pm 0.13^{*}$ & $5.14 \pm 0.13^{\wedge}$ \\
\hline HDL cholesterol (mmol/l) & $1.25 \pm 0.04$ & $1.26 \pm 0.04$ & $1.11 \pm 0.03^{* * * \wedge \wedge}$ \\
\hline LDL-cholesterol (mmol/l) & $3.18 \pm 0.12$ & $2.90 \pm 0.11^{* * *}$ & $3.18 \pm 0.12^{\wedge}$ \\
\hline Atherogenic index LDL/HDL & $2.64 \pm 0.13$ & $2.38 \pm 0.12^{*}$ & $3.18 \pm 0.12^{* * * \wedge \mu}$ \\
\hline C-reactive protein $(\mathrm{mg} / \mathrm{l})$ & $1.91 \pm 0.22$ & $1.71 \pm 0.21$ & $1.99 \pm 0.25$ \\
\hline Homocysteine $(\mu \mathrm{mol} / 1)$ & $14.2 \pm 0.8$ & $11.4 \pm 0.6^{* * *}$ & $10.4 \pm 0.5^{* * *}$ \\
\hline Methionine $(\mu \mathrm{mol} / \mathrm{l})$ & $29.3 \pm 1.1$ & $22.9 \pm 0.7^{* * *}$ & $22.5 \pm 0.8^{* * *}$ \\
\hline Vitamin B12 (pmol/l) & $249 \pm 13$ & $294 \pm 12^{* * *}$ & $307 \pm 13^{* * *}$ \\
\hline Vitamin B6 $(\mu \mathrm{g} / \mathrm{l})$ & $9.50 \pm 0.5$ & $6.35 \pm 0.8^{*}$ & $8.83 \pm 0.7^{\wedge}$ \\
\hline Cysteine $(\mu \mathrm{mol} / \mathrm{l})$ & $126 \pm 2$ & $116 \pm 3^{* * *}$ & $119 \pm 2^{* * *}$ \\
\hline Asymmetric dimethyl-arginine $(\mathrm{nmol} / \mathrm{ml})$ & $1.00 \pm 0.03$ & $0.46 \pm 0.03^{* * *}$ & $0.87 \pm 0.02^{* * \wedge \mu}$ \\
\hline
\end{tabular}

The results are expressed as the mean \pm SEM

* (2), (3) vs. (1) * $\mathrm{p}<0.05 * * \mathrm{p}<0.01 * * * \mathrm{p}<0.001$

$\wedge$ (3) vs. (2)^ $\mathrm{p}<0.05^{\wedge \wedge \wedge} \mathrm{p}<0.001$ 
and protects the myocardium from ischemic damage. It has no clear effects on serum lipid profile and on insulin resistance. The metaanalysis of epidemiological studies showed an inverse association between flavonol intake and coronary heart disease and stroke.

An increased selenium intake can decrease cardiovascular risk by reduction of lipid peroxidation, inflammation inhibition or by improvement of blood lipid spectrum (5).

A high content of polyphenols was observed in curcuma. Alcoholic curcuma extract has anti-inflammatory, immunomodulative, anticarcinogenic and antisclerotic effects (6). Curcuma extract in dose of $200 \mathrm{mg}$ consumed in duration of 45 days significantly decreased lipid peroxide concentration in healthy men aged 27-67 years (7). In other study, a similar dose of curcuma caused the reduction of HDI- and LDL- peroxides by $25-50 \%$ (8).

Literature data described above suppors the finding of an improved lipid spectrum by biscuits consumption (Tab. 2). The levels of total and LDL-cholesterol as well as values of the atherogenic index were significantly reduced after a 2-month biscuits consumption.

A high sensitivity C-reactive protein (hsCRP), a marker of inflammation, confers additional prognostic value at all levels of cholesterol, Framingham coronary risk score, severity of the metabolic syndrome, blood pressure and in those with and without subclinical atherosclerosis (9). The subjects with predominant consumption of plant food have significantly reduced values of hsCRP vs. general population $(10,11)$ as a consequence of sufficient and regular consumption of fruit and vegetables, which are the rich sources of salicylates and other anti-inflammatory compounds (12). These compounds are contained in onion and curcuma also. A non-significantly reduced hs-CRP concentration after biscuits consumption was recorded (Tab. 2).

During the last decade, several observational studies about homocysteine ( $\mathrm{HCy})$ as a predictor for atherosclerosis risk showed that the overall risk for vascular disease is small. In prospective longitudinal studies, a weak association between HCy and atherothrombotic vascular disease was reported, compared to retrospective case-control and cross-sectional studies with a stronger association (13). In spite of this fact, for the disease prevention is important that all atherogenic markers were in normal values. The significant reduction of HCy was found in onion biscuits consumption (Tab. 2). Remethylation pathway of HCy degradation dependent on nutritional determinants vitamin B12 and folic acid prevails at lower methionine concentration. Folic acid in subject blood was significantly reduced during biscuits consumption probably as a consequence of low consumption of folic acid rich food in late autumn and winter. Transsulfuration pathway of HCy degradation is non-dominant because the significantly reduced cysteine and vitamin B6 concentrations were found. An alternative pathway for methylation of $\mathrm{HCy}$ provided by betaine - HCy methyltransferase using betaine as methyl donor is probably the sole argument for reduced HCy value (14). The biscuits were produced from whole grain flour with a higher content of betaine (15). After a two month exclusion of biscuit vs. the end of consumption, the concentrations of $\mathrm{HCy}$, methionine, cysteine and folic acid were not changed. The similarly lower HCy con- centration after the washout period is perhaps a consequence of short-term period after the end of consumption.

A novel cardiovascular risk factor - asymmetric dimethylarginine ADMA (16) is an endogenous inhibitor of nitric oxide (NO) associated with an increased risk of cardiovascular disease. The methyl groups in dimethylarginine molecules are derived from S-adenosylmethionine, an intermediate in the Hcy-methionine pathway (17). The major functional abnormality underlying hyperhomocysteinemia-induced endothelial dysfunction appears to be related to decreased bioavailability of endothelium-derived NO. $\mathrm{NO}$ is a potent vasodilator that is produced by endothelial nitric oxide synthase. The mechanisms, by which hyperhomocysteinemia decreases the bioavailability of endothelium-derived NO, are not completely understood and may be multifactorial (18). One mechanism for endothelial dysfunction during hyperhomocysteinemia may be an oxidative inactivation of $\mathrm{NO}$ to form toxic peroxynitrite (19). Evidence for oxidative stress during hyperhomocysteinemia has been obtained in many animal models (18). Elevation of the ADMA may be a key mediator of decreased NO bioavailability during hyperhomocysteinemia. ADMA was significantly reduced by biscuits consumption (Tab. 2). A decreased ADMA value at actually reduced $\mathrm{HCy}$ concentration after biscuits consumption may be explained by introduced relationship between HCy and ADMA.

During the washout period, the values of total-cholesterol, LDL-cholesterol, atherogenic index, vitamin B6, hs-CRP and ADMA completely or non-completely returned to levels before consumption.

\section{References}

1. Contor L. Functional food science in Europe. Nutr Metab Cardiovasc Dis 2001; 11: 20-23.

2. Melnyk S, Pogribna M, Pogribny I, Hine RJ, James SJ. A new HPLC method for the simultaneous determination of oxidized and reduced plasma aminothiols using coulometric electrochemical detection. J Nutr Biochem 1999; 10: 490-497.

3. Erkkila A, DeMello VD, Riserus U, Laaksonen DE. Dietary fatty acids and cardiovascular disease, an epidemiological approach. Progr Lipid Res 2008; 47: 172-187.

4. Perez-Vizcairo F, Duarte J. Flavonols and cardiovascular disease. Mol Aspects Med 2010; 31: 478-494.

5. Ravn-Haren G, Bugel S, Krath, B N, Hoac T, Stagsted J, Jorgensen K, Bresson JR, Larsen EH, Dragsted LO. A short-term intervention trial with selenate, selenium enriched yeast and selenium enriched milk: Effects on oxidative defence regulation. Br J Nutr 2008; 99: 883-892.

6. Miquel J, Bernd A, Sempere JM, Díaz-Alperi J, Ramírez A. The curcuma antioxidants: pharmacological effects and prospects for future clinical use.Arch Gerontol Geriat 2002; 34: 37-46.

7. Ramírez-Boscá A, Soler A, Carrión-Gutiérez MA, Laborda-Alvarez A, Quintanilla-Almagro E. Antioxidant curcuma extracts decrease the blood lipid peroxide levels of human subjects. Age 1995; 18: 207-210.

8. Ramírez-Boscá A, Carrión-Gutiérez MA, Soler A, Puerta C, Diáz A, Quintanilla E, Bernd A, Miquel J. Effects of the antioxidant turmeric on lipoprotein peroxides: implication for the prevention of atherosclerosis. Age 1997; 20: 165-168. 
9. Bassuk SS, Rifai N, Ridker PM. High sensitivity C-reactive protein, clinical importance. Curr Probl Cardiol,2004; 29: 439-493.

10. Krajcovicova-Kudlackova M, Blazicek P. C-reactive protein and nutrition. Bratislava Med J 2005; 106: 345-347.

11. Szeto ZT, Kwok TC, Benzie IF. Effect of a long-term vegetarian diet on biomarkers of antioxodant status and cardiovascular disease risk. Nutr 2004; 20: 863-866.

12. Lawrence JR, Peter R, Baxter GJ. Urinary excretion of salicyluric and salicylic acids by non-vegetarians, vegetarians and patients taking low dose aspirin. J Clin Pathol 2003; 56: 649-650.

13. Kaul S, Zadeh AA, Shah PK. Homocysteine hypothesis for atherothrombotic cardiovascular disease, not validated. J Am Coll Cardiol 2006; 48: 914-923.

14. Krajčovičová-Kudláčková $M$, Blažíček P. Effect of nutrition at homocysteine levels. Biologia 2002; 57: 395-399.
15. Zeisel SH, Mar HM, Howe JC, Holden JM. Concentrations of choline-containing compounds and betaine in common foods. J Nutr 2003; 133: $1302-1307$.

16. Anderssohn M, Schwedhelm E, Luneburg N, Vasan RS, Boger RH. Asymmetric dimethylarginine as a mediator of vascular dysfunction and a marker of cardiovascular disease and mortality, an intriguing intercation with diabetes mellitus. Diab Vasc Dis Res 2010; 7: 105-118.

17. loger RH. Association of asymmetric dimethylarginine and endothelial dysfunction. Clin Chem Lab Med 2003; 41: 1467-1472.

18. Dayal S, Lentz SR. ADMA and hyperhomocysteinemia. Vasc Med 2005; 10: 27-33.

19. Forstermann U. Nitric axide and oxidative stress in vascular disease. Pflug Arch 2010; 459: 923-939.

Received March 9, 2011. Accepted August 18, 2012. 